\title{
Knockdown of GATAD2A suppresses cell proliferation in thyroid cancer in vitro
}

\author{
ZONGPING WANG ${ }^{*}$, JIE KANG ${ }^{*}$, XIANZHAO DENG, BOMIN GUO, BO WU and YOUBEN FAN \\ Department of General Surgery, The Sixth People's Hospital Affiliated to \\ Shanghai Jiaotong University, Shanghai 200233, P.R. China
}

Received August 17, 2016; Accepted January 4, 2017

DOI: $10.3892 /$ or.2017.5436

\begin{abstract}
GATAD2A (GATA zinc finger domain containing $2 \mathrm{~A}$ ), is a subunit of NuRP (nucleosome remodeling and histone deacetylation) which plays key roles in tumor growth inhibition and embryonic development. However, its role in thyroid cancer remains unclear. In our study, we established two thyroid cancer cell lines by lentivirus-delivered short hairpin (shRNA) to knockdown the expression of GATAD2A. Then loss-of-function assays indicated that knockdown of GATAD2A decreased the ability of cell proliferation and colony formation in thyroid cancer cells by MTT and colony formation assay, respectively. Moreover, cell cycle assay by flow cytometry revealed that the percentage of cells in G0/G1 phase was significantly decreased in GATAD2A knockdown cells accompanied by increase of cells in G2/M phase. Furthermore, inhibition of GATAD2A promoted cell apoptosis via elevating the expression of caspase- 3 and PARP cleavage using Annexin V/7-AAD double staining and western blotting. In conclusion, GATAD2A is an essential factor in thyroid cancer cell growth and apoptosis, and may be a potential therapeutic biomarker in thyroid cancer.
\end{abstract}

\section{Introduction}

Thyroid cancer is a common endocrine malignancy and ranked the ninth most frequent in the USA in 2014 with estimated 62,980 new patients with thyroid carcinoma in the USA in 2014. Over time, the incidence and morbity rates were increased by $5.5 \%$ and $0.8 \%$, respectively each year $(1,2)$. The incidence of thyroid cancer is the sixth highest in women and

Correspondence to: Dr Bo Wu or Youben Fan, Department of General Surgery, The Sixth People's Hospital Affiliated to Shanghai Jiaotong University, 600 Yi-San Road, Shanghai 200233, P.R. China E-mail: shthyroid@126.com

E-mail: lett_thyroid@163.com

*Contributed equally

Key words: thyroid cancer, GATAD2A, cell proliferation, cell apoptosis more than three-fold in women compared to men (3). Thyroid cancer is classed into three categories: MTC (medullary thyroid cancer), DTC (differentiated thyroid cancer, include papillary thyroid cancer, follicular thyroid cancer and poorly differentiated thyroid cancer) and ATC (anaplastic thyroid cancer) $(3,4)$. MTC is originated from the parafollicular $\mathrm{C}$-cells and the remaining subtypes are derived from thyroid follicular cells $(1,5,6)$. Fine-needle aspiration (FNA) biopsy is a useful clinical tool to examine thyroid cancer, but the accuracy of diagnosis still need improvement (7). Radiotherapy and chemotherapy are effective but the appropriate selection and dosage are still controversial (6). Currently, molecular analysis provides better understanding of the development and progression of thyroid cancer.

Transcription is regulated through various chromatinremodeling complexes such as NuRD (nucleosome remodeling and histone deacetylation) complex. GATAD2A (GATA zinc finger domain containing 2A), is a subunit of NuRD complex, which comprises HDAC1/2 (histone deacetylases), CHD3/4 (ATP-dependent remodeling enzymes), RbAp46/48 (histone chaperones) and MTA1/2/3 (specific DNA-binding proteins) (8). The NuRD complex is a key factor in various biological progresses, including tumor growth inhibition, cellular differentiation, embryonic development and the general repression of transcription $(8,9)$. GATAD2B is a paralog of GATAD2A (10). GATAD2A/B have two conserved regions known as $\mathrm{CR} 1$ and $\mathrm{CR} 2$ which could bind to MBD3 (methyl-CpG binding domain protein 3), a key protein of the NuRD $(10,11)$. It is demonstrated that the GATAD2A plays essential roles in early mouse development (12). However, little information is known regarding its biology in thyroid cancer up to now.

In this study, thyroid cancer cell lines (Cal-62 and 8305C) were used to detect the expression of GATAD2A by qPCR and western blotting. The effects of GATAD2A on Cal-62 and $8305 \mathrm{C}$ proliferation, cell cycle and apoptosis were analyzed. Moreover, we detected the expression of apoptosis-associated proteins, caspase- 3 and PARP. These results might help to diagnose the thyroid cancer and improve the targeted therapies.

\section{Materials and methods}

Cell culture. The cell lines (Cal-62, 8305C and 293T) were obtained from Cell Bank of Chinese Academy of Science 
(Shanghai, China). Cal-62, a thyroid cancer cell line, was cultured in DMEM supplemented with 10\% FBS (fetal bovine serum, Biowest, S1810). 8305C, a thyroid cancer cell line, was cultured in EMEM containing 10\% FBS, 1\% L-Glu (21127022, Gibco, Cambrex, MD, USA) and 1\% NEAA. 293T cell line was also cultured in DMEM containing 10\% FBS (fetal bovine serum, Biowest, S1810). All cell lines were maintained at $37^{\circ} \mathrm{C}$ in an incubator (311, Thermo) with $5 \% \mathrm{CO}_{2}$.

Construction of GATAD2A knockdown lentivirus. According to the sequence of GATAD2A, two vectors shRNA S1 and shRNA S2 were designed. The sequences are as follows: shRNA S1: 5'-CGTGCTGAAGCAGGTCATAAACTCGAG TTTATGACCTGCTTCAGCACGTTTTT-3'; shRNA S2: 5'-CAGAACCTACTGGAGACACAACTCGAGTTGTGTCT CCAGTAGGTTCTGTTTTT-3'; control shRNA: 5'-GCGG AGGGTTTGAAAGAATATCTCGAGATATTCTTTCAAA CCCTCCGCTTTTTT-3'. The three shRNAs were cloned into the plasmid pFH-L (Shanghai Hollybio, China). Recombinant lentiviruses were generated by transfection of $80 \% 293 \mathrm{~T}$ cells with lentivirus (including controls) and the helper plasmids including pVSVG-I and pCMVAR8.92 (Shanghai Hollybio, China). After filtered and determined the viral titers, 10 and 8 MOI (multiplicities of infection) were achieved in Cal-62 and $8305 \mathrm{C}$ cell lines, respectively. After infected for 96 and $72 \mathrm{~h}$, the achieved infection efficiency was $80 \%$, and the expression of GATAD2A was analyzed by RT-qPCR and western blotting.

Quantitative real-time PCR (RT-qPCR). After infected with lentivirus for 5 days, total RNAs of cultured cells (Cal-62 and 8305C) were extracted using TRIzol (15596-018, Invitrogen, Carlsbad, CA, USA). The mRNA level of GATAD2A was evaluated by RT-qPCR using the $\mathrm{iQ}^{\mathrm{TM}}$ SYBR Green Supermix kit (1708880, Bio-Rad, Hercules, CA, USA) on Bio-Rad Connet Real-time PCR platform. The $20 \mu \mathrm{l}$ mixture reaction system contained $2 X$ SYBR Premix Ex Taq $10 \mu 1$, cDNA $5 \mu \mathrm{l}$, forward and reverse primers $(2.5 \mu \mathrm{M}) 0.5 \mu \mathrm{l}$, and $\mathrm{ddH}_{2} \mathrm{O} 4.5 \mu \mathrm{l}$. Actin was applied as the input reference. Primers were designed based on cDNA to amplify GATAD2A (NM_017660.3) and actin. The primers used were as follows: GATAD2A: Forward, 5'-TGCTCGCCTCCTGCCTGTAG-3'; Reverse, 5'-GACACAAAGCCAAGCCAGACC-3'; actin: Forward, 5'-GTGGACATCCGCAAAGAC-3'; Reverse, 5'-AAAGGGTGTAACGCAACTA-3'. The mRNA was determined by using the $2^{-\triangle \Delta C T}$ method.

Western blotting. After infection for 6 days, cells were collected, washed with PBS and lysed in protein lysate (2X SDS Sample Buffer (100 mMTris-HCl (pH 6.8), 10 mM EDTA (Sangon, Shanghai, China), 4\% SDS (SB0485-500 g, Sangon), $10 \%$ glycine). Total protein was measured through BCA method and separated by $10 \%$ SDS-PAGE (45 $\mu \mathrm{g} /$ lane). Then proteins were transferred to PVDF (polyvinylidene difluoride) membranes and blocked in 40-50 ml TBST (Trisbuffered saline containing $0.1 \%$ Tween-20) including 5\% skim milk for $1 \mathrm{~h}$ at room temperature. The membranes were then incubated with primary antibodies (rabbit anti-GATAD2A, Abgent, \#20258a, 1:500; rabbit anti-PARP, Cell Signaling, \#9542, 1:1000; rabbit anti-caspase-3, Cell Signaling, \#9661,
1:500; rabbit anti-GAPDH antibody, Proteintech, 10484-1-AP, 1:500000) at $4^{\circ} \mathrm{C}$ overnight. The membranes were then washed three times with TBST for $10 \mathrm{~min}$ and probed with secondary antibody (goat anti-rabbit HRP, Santa Cruz Biotechnology, SC-2054, dilution: 1:5000) for $2 \mathrm{~h}$ at room temperature. After three washes with TBST, the membranes were visualized using the ECL Plus Western Blotting System kit (Amersham, RPN2132), and the chemiluminescence was detected using $\mathrm{X}$-ray film (Kodak).

MTT proliferation and colony formation assays. Cal-62 and $8305 \mathrm{C}$ cell lines were infected for 96 and $120 \mathrm{~h}$, respectively, the inoculation density of cell lines was adjusted to $2 \times 10^{3}$ per well of Cal-62 and $5 \times 10^{3}$ per well of $8305 \mathrm{C}$ in 96 -well plates, respectively. After cell adhesion, $20 \mu \mathrm{l}$ MTT $(5 \mathrm{mg} / \mathrm{ml}$, \#M2128, Sigma, St. Louis, MO, USA) was added and cultured for $4 \mathrm{~h}$. Then, $100 \mu \mathrm{l}$ acidified isopropanol (10\% SDS, 5\% isopropanol and $0.01 \mathrm{~mol} / 1 \mathrm{HCl}$ ) was added to stop the reaction and OD was determined on microplate reader.

For evaluation of colony formation, the Cal-62 cell lines were infected for $96 \mathrm{~h}$ and the density was achieved to 600 cells per well in 6-well plate. After cultured for 7 days, the cells were fixed by $700 \mu \mathrm{l} 4 \%$ paraformaldehyde per well. The plates were washed with PBS (phosphate-buffered saline) twice and stained with $700 \mu \mathrm{l}$ crystal purple per well for $5 \mathrm{~min}$. After washed with PBS and air-dried, the number of colonies was manually counted under the microscope.

Determination of cell cycle by flow cytometry. After infection for 6 and 8 days, the Cal-62 and 8305c cell lines were seeded in $6-\mathrm{cm}$ dishes at $2 \times 10^{5}$ cells and $8 \times 10^{4}$ cells per well, respectively. The CAL-62 cells were cultured for 6 days and harvested when the density reached $80 \%$. The collected cells were fixed by $75 \%$ cold ethanol (10009269, Sinopharm Chemical Regant Co. Ltd., Shanghai, China) for 1 day at $4^{\circ} \mathrm{C}$, the cells were washed with cold PBS and stained with $500 \mu \mathrm{l}$ PI (Propidium Iodide) buffer (C1052, Beyotime Biotechnology, Shanghai, China) for $1 \mathrm{~h}$ at $37^{\circ} \mathrm{C}$ in the dark. The cell cycles were analyzed by flow cytometry at $24 \mathrm{~h}$.

Determination of cell apoptosis by flow cytometry. Apoptosis of Cal-62 and 8305C cell lines were detected using Annexin V/7-AAD double staining kit (KGA1026, Nanjing KeyGen Biotech. Co., Ltd.) after lentivirus infection for 6 and 8 days, respectively. When cells $\left(5 \times 10^{5}\right.$ cells per dish) were cultured in $10-\mathrm{cm}$ dishes to reach $80 \%$ confluency, they were harvested, washed twice with PBS, and stained with $5 \mu 1$ Annexin V-APC and $5 \mu 1$ 7-AAD for 5-15 min. Then cells were kept on ice in the dark and subjected to apoptosis analysis on flow cytometry.

Statistical analysis. All the experiments were repeated three times. The SPSS 17.0 was used to analyze the results with t-test or one-way ANOVA. The results were considered to be statistically significant at P-value $<0.05$.

\section{Results}

Inhibition of GATAD2A in Cal-62 and 8305C cell lines. To investigate the function of GATAD2A in thyroid cancer, 

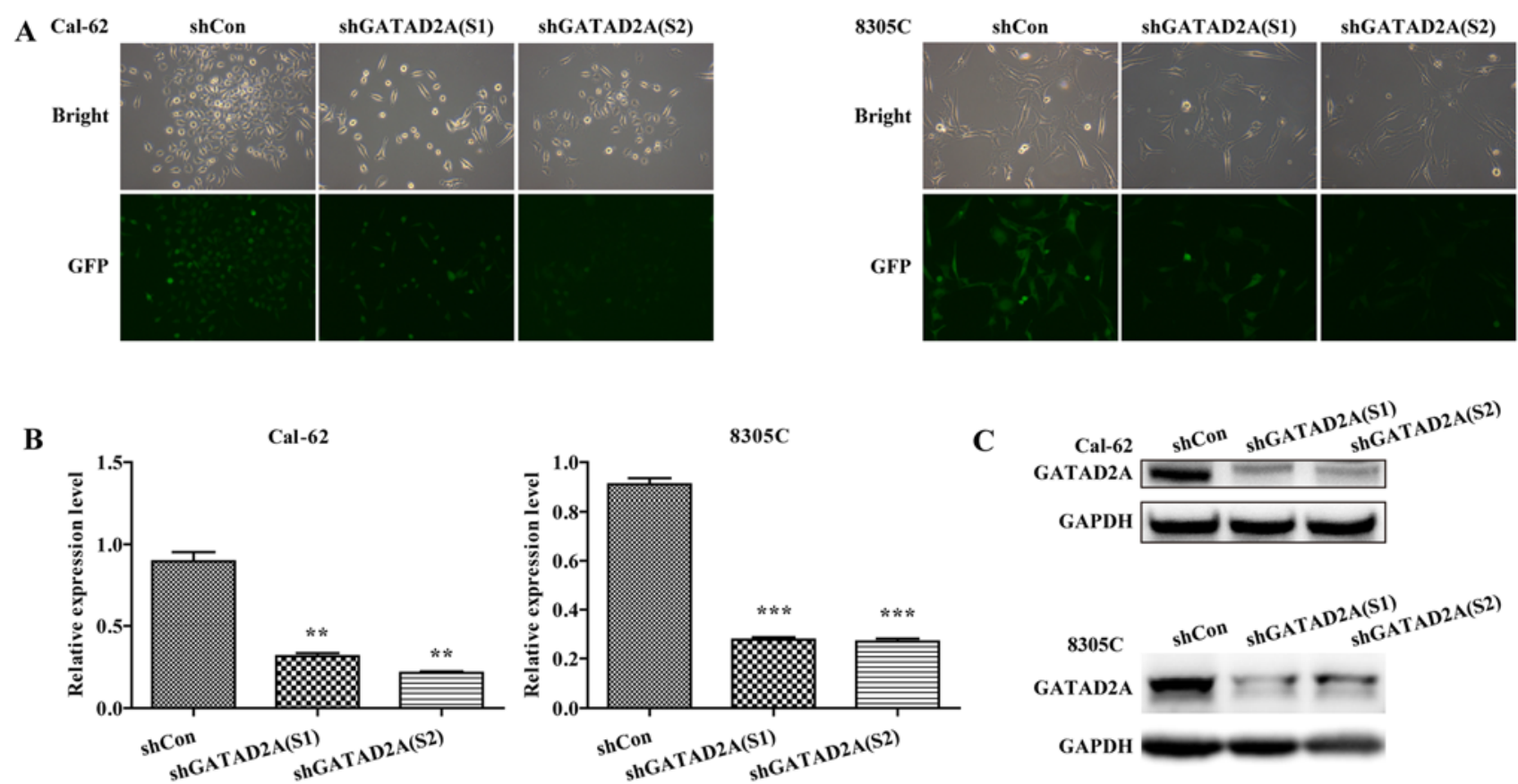

Figure 1. Knockdown efficiency of GATAD2A by lentivirus infection in the thyroid cancer cell lines, Cal-62 and $8305 \mathrm{C}$. (A) Microscopic images of Cal-62 and 8305C cells infected with lentivirus. (B) RT-PCR analysis of GATAD2A knockdown efficiency in Cal-62 and 8305C cells. (C) Western blot analysis validated the knockdown efficiency of GATAD2A in Cal-62 and 8305C cells. ${ }^{* *} \mathrm{P}<0.01,{ }^{* * * *} \mathrm{P}<0.001$; Scale bars, $10 \mu \mathrm{m}$.
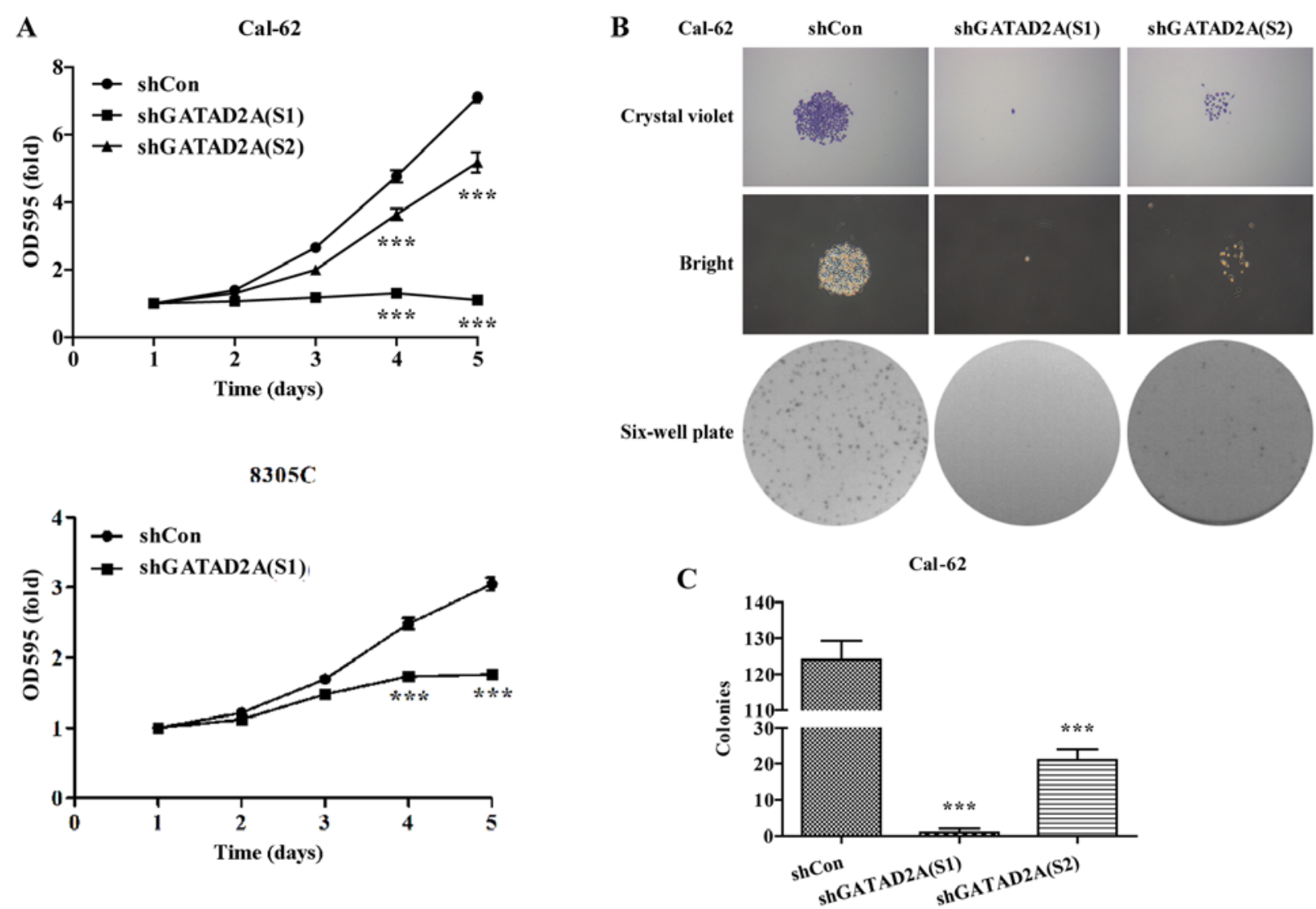

Figure 2. Downregulation of GATAD2A inhibits the proliferation and colony formation abilities of thyroid cancer cells. (A) MTT assay was used to determine cell viability in Cal-62 and 8503C cells after GATAD2A knockdown. (B) Representative microscopic images of colonies were stained by crystal violet in Cal-62 cells. (C) Statistical analysis of the number of colonies in Cal-62 cells. ${ }^{* * *} \mathrm{P}<0.001$; Scale bars, $25 \mu \mathrm{m}$.

the expression of GATAD2A was inhibited by infection with shGATAD2A (S1) or shGATAD2A (S2) in Cal-62 and
8305C cell lines. We further examined the expression level of GATAD2A by RT-PCR and western blotting in shGATAD2A 
$\mathbf{A}$

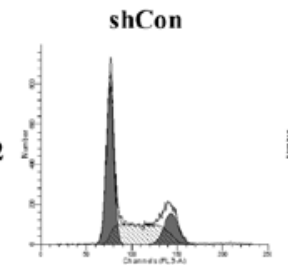

ShGATAD2A(S1)
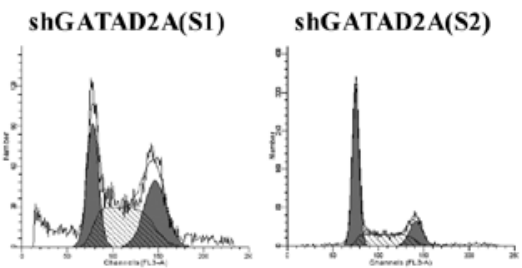

Cal-62
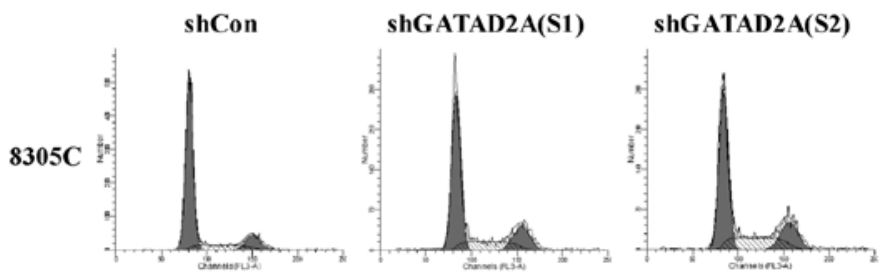

$8305 \mathrm{C}$

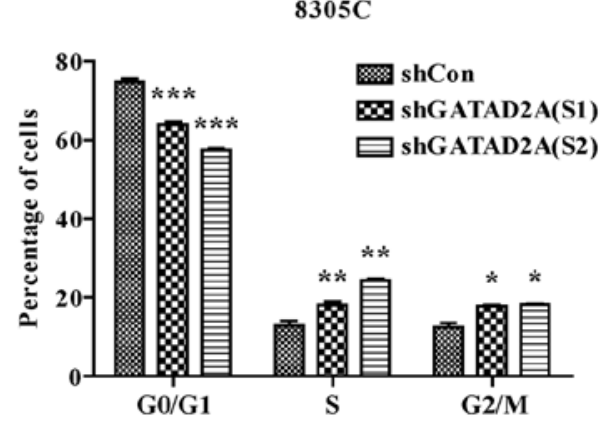

$8305 \mathrm{C}$

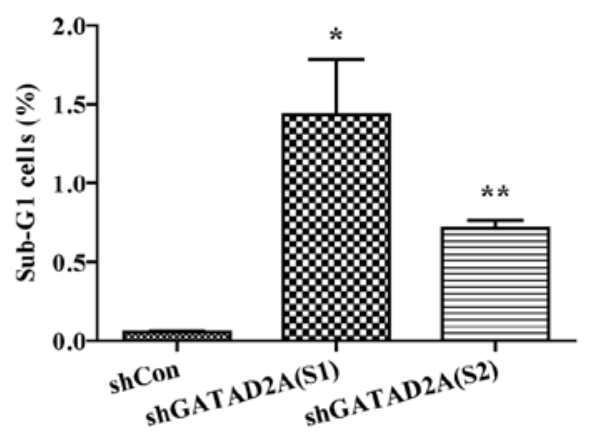

Figure 3. Depletion of GATAD2A arrests cell cycle progression in Cal-62 and 8305C cell lines. (A) Cell cycle progression was analyzed by flow cytometry. (B) Statistical analysis of the percentage of cells in G0/G1, S and G2/M phases in Cal-62 and 8305C cells after GATAD2A knockdown. (C) Statistical analysis of the percentage of cells in sub-G1 phase in Cal-62 and 8305C cells after GATAD2A knockdown. ${ }^{*} \mathrm{P}<0.05,{ }^{* *} \mathrm{P}<0.01,{ }^{* * *} \mathrm{P}<0.001$.

and shCon infected cell lines. As shown in Fig. 1A, most cells presented GFP-positive expression under a microscope, suggesting higher infection efficiency. Furthermore, the mRNA levels of GATAD2A were significantly downregulated in shGATAD2A ( $\mathrm{S} 1$ and $\mathrm{S} 2$ ) compared to the shCon group in Cal-62 and $8305 \mathrm{C}$ cell lines (Fig. $1 \mathrm{~B}, \mathrm{P}<0.01, \mathrm{P}<0.001$ ). Consistently, the shGATAD2A (S1 and S2) obviously downregulated the protein level of GATAD2A in Cal-62 and 8305C cells (Fig. 1C). There was a difference in the expression levels of S1 and S2 in Cal-62 cells, but the difference is not significant. Collectively, we successfully constructed GATAD2A-silencing Cal-62 and 8305C cell lines.

Effects of GATAD2A on cell proliferation and colony formation. The potential effects of GATAD2A on cell proliferation and colony formation were explored in Cal-62 and $8305 \mathrm{C}$ cell lines. The same number of CAL-62 and $8305 \mathrm{C}$ cells from shGATAD2A and shCon group was subjected to MTT assay. The results showed that Cal-62 and $8305 \mathrm{C}$ cells presented significantly slower proliferative rate $(\mathrm{P}<0.001$, Fig. $2 \mathrm{~A})$ after transfection with shGATAD2A (S1) or shTAGAD2A (S2). Notably, the suppressive effects in Cal-62 cells were more obvious than that in $8305 \mathrm{C}$ cells.
To further determine the effects of GATAD2A on cell proliferation, we chose Cal-62 cells to perform colony formation assay. As shown in Fig. 2B, The size was relatively smaller and colonies were fewer in shGATAD2A (S1) or shGATAD2A (S2) groups compared with shCon groups in Cal-62 cells. Statistical analysis further confirmed that knockdown of GATAD2A significantly reduced colonies formed in Cal-62 cells (Fig. 2C, P<0.001). Notably, Cal-62 cells treated with shGATAD2A (S1) presented more obvious growth inhibition than shGATAD2A (S2). The above results revealed that GATAD2A might play an important role in thyroid cancer cell growth and proliferation.

Effects of GATAD2A on cell cycle progression. To determine the underlying mechanism of inhibition of cell proliferation, cell cycle was analyzed by flow cytometry. The percentage of cell distribution was observed as significantly different in shGATAD2A and Con groups (Fig. 3A). Compared to shCon, the percentage of cells in G0/G1 phase was significantly decreased, while the cells in G2/M phase were remarkably increased in shGATAD2A infected Cal-62 and 8305C cell lines (Fig. 3B, P<0.001), indicating cell cycle was arrested in $\mathrm{G} 2 / \mathrm{M}$ phase by GATAD2A knockdown. In addition, 
A

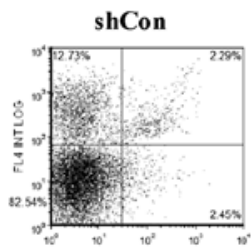

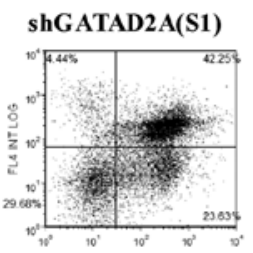
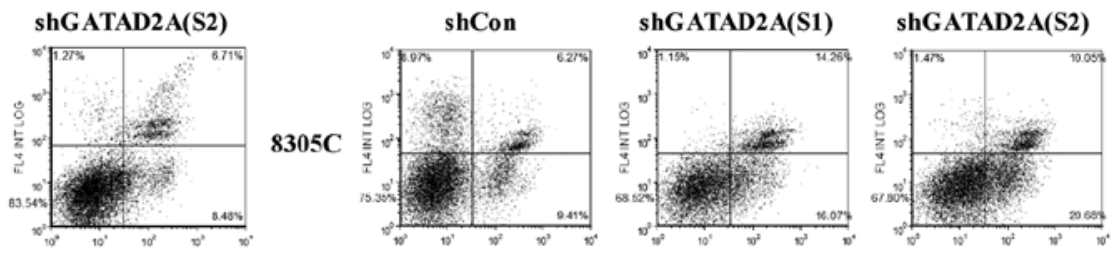

B
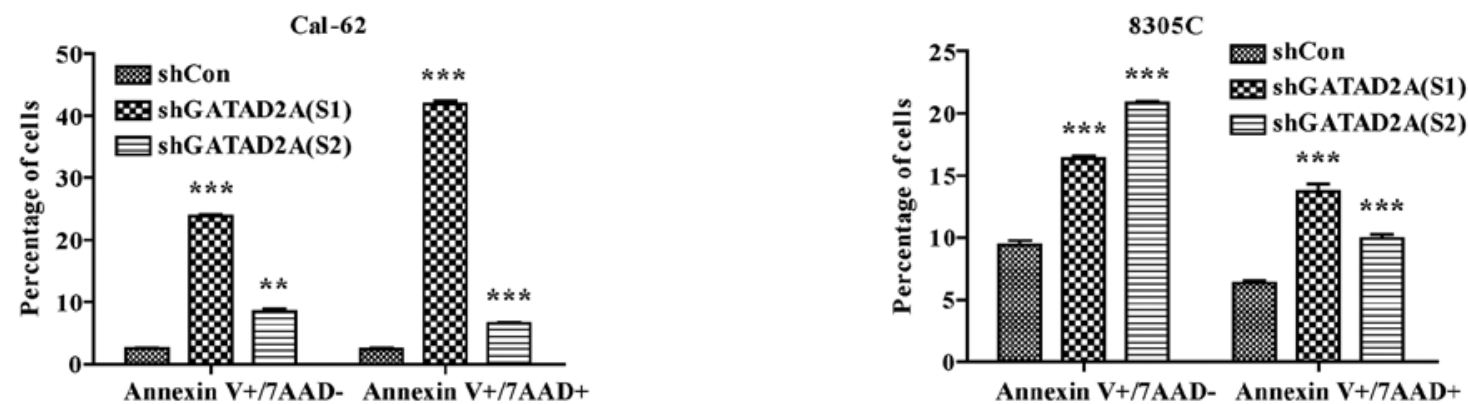

Figure 4. Depletion of GATAD2A promoted cell apoptosis including early-stage (Annexin $\mathrm{V}^{+} / 7-\mathrm{ADD}^{-}$) and late-stage (Annexin $\left.\mathrm{V}^{+} / 7-\mathrm{ADD}^{+}\right)$. (A) Flow cytometry combined with Annexin V/7-AAD double staining was used to analyze cell apoptosis in Cal-62 and 8305C cells after GATAD2A knockdown. (B) Statistical analysis of apoptotic cell rate in Cal-62 and 8305C cells after GATAD2A knockdown. ${ }^{* * *} \mathrm{P}<0.001$.

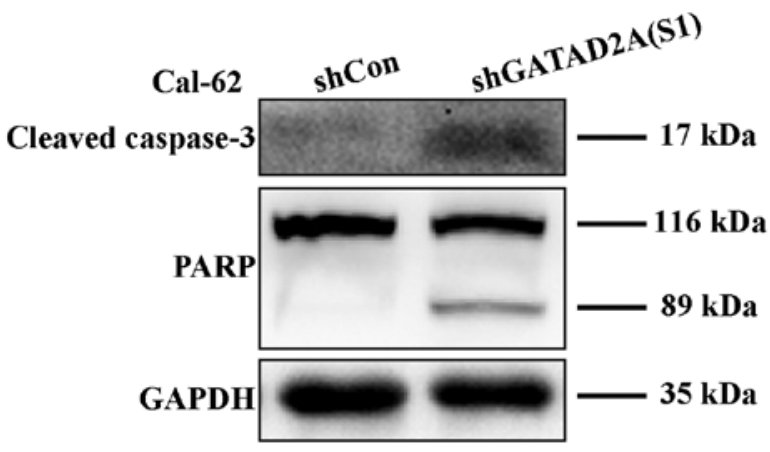

Figure 5. Knockdown of GATAD2A upregulated apoptotic markers caspase-3 and PARP cleavage in Cal-62 cells by western blot analysis. GAPDH was used as internal control.

the sub-G1 cell number of both cell lines infected with shGATAD2A was significantly increased (Fig. 3C, $\mathrm{P}<0.05$, $\mathrm{P}<0.01, \mathrm{P}<0.001)$. Taken together, these data suggested that knockdown of GATAD2A-suppressed cell proliferation might be via cell cycle arrest.

Effects of GATAD2A on cell apoptosis. In addition, we further investigated the effects of GATAD2A knockdown on cell apoptosis by flow cytometry (Fig. 4A). As shown in Fig. 4B, the number of apoptotic cells in shGATAD2A infected Cal-62 and $8305 \mathrm{C}$ cell lines was significantly higher than that in shCon group $(\mathrm{P}<0.001)$. Based on the above data, we observed there existed differences in suppressing cell proliferation and inducing cell cycle arrest and apoptosis between S1 and S2. Overall, functional experiments revealed shGATAD2A (S1) was more powerful than shGATAD2A (S2) in suppressing cell proliferation, induced cell cycle arrest and apoptosis. Therefore, shGATAD2A (S1) was chosen to study the effect of GATAD2A in regulating the apoptosis-related proteins in Cal-62 cells. In line with flow cytometry, caspase-3 cleavage was higher in shGATAD2A (S1) infected cells and its substrate PARP (poly ADP-ribose polymerase), a marker of apoptosis and activation of caspase-3, was also elevated compared to the control group (Fig. 5). The results suggested that GATAD2A plays essential roles in cell proliferation of thyroid cancer.

\section{Discussion}

It has been implicated that NuRD participates in the regulation of transcriptional events, chromatin assembly, cell cycle progression and genetic instability (13). Depending on cell types, the NuRD complex plays roles in both promoting and suppressing tumorigenesis (13). It has been reported that GATAD2A is an important structural subunit of the NuRD complex and is directly associated with histone tails $(11,14)$. The amino-terminal conserved region of GATAD2A directly interacts with MBD2 or MBD3 and the C-terminal conserved region can interact with histone tails and is essential for targeting to the specific genomic loci $(11,14)$. This evidence suggests GATAD2A might be closely associated with tumorigenesis. Thus, our study revealed the close relationship between GATAD2A and thyroid cancer progression. Functional analysis demonstrated that knockdown of GATAD2A suppressed thyroid cancer cell growth and proliferation.

To better understand the underlying mechanism of cell growth inhibition, the distribution of cell cycle phases was analyzed in thyroid cancer cells after GATAD2A knockdown. As expected, knockdown of GATAD2A resulted in G2/M arrest in the cell cycle. A previous study showed NuRD complex is an active regulator of both the G1/S and the G2/M checkpoints and its defection affects checkpoint control of G2/M through disruption in histone modification (13). It might indicate that GATAD2A is the key regulator in G2/M check point through interaction with other partners within the NuRD complex.

Moreover, the flow cytometry results revealed that the abnormal cell group distributed in the sub-G1 phase. Sub-G1 
peak was defined as the cells with deficit in DNA content and the DNA fragments was stained (15-17). DNA fragmentation is the hallmark of cell apoptosis $(18,19)$. Quantification of the percentage of apoptotic cells in sub-G1 phase is commercially available to detect cell apoptosis. However, the sub-G1 DNA content cannot be used as a sole marker of apoptotic cells. Further, we detected cell apoptosis by flow cytometry and the results revealed that knockdown of GATAD2A increased cell apoptosis in both early and late stages compared to the control group. These results kept in line with the results from the previous assay, which indicated that GATAD2A played potential roles in thyroid cancer cell proliferation.

In apoptosis progress, caspase- 3 is a crucial mediator of apoptosis and cleaved to activate (20). Cleaved caspase-3 displayed upregulation in the GATAD2A knockdown group. Moreover, activation of PARP is a response to DNA damaging compounds and acts as a target of caspase-3 for degradation. The PARP was cleaved into two polypeptide fragments and the subsequent loss in its activity accompanies apoptosis (21-23). In addition, it has been shown that the NuRD complex is rapidly recruited to the sites of DSBs (double-strand breaks) through the activity of the poly(ADP ribose) polymerase (PARP) $(24,25)$. It might indicate that GATAD2A knockdown is the executor response to DNA damage and active downstream cascade of cell death.

Based on these data, we could conclude that suppression of GATAD2A attenuated thyroid cancer cell proliferation and colony formation and promoted apoptosis, through activating the caspase- 3 and PARP. To further explore its clinical significance, more work is still required to validate the findings of the current study in terms of a clinical application, due to the low number of clinical specimens examined.

\section{References}

1. Weitzman SP and Cabanillas ME: The treatment landscape in thyroid cancer: A focus on cabozantinib. Cancer Manag Res 7: 265-278, 2015

2. Grande $\mathrm{C}$ and Brose MS: The evolving treatment landscape for metastatic differentiated thyroid cancer. J Adv Pract Oncol 5: 461-465, 2014.

3. LiVolsi VA: The pathology of autoimmune thyroid disease: a review. Thyroid 4: 333-339, 1994.

4. Aschebrook-Kilfoy B, Ward MH, Sabra MM and Devesa SS: Thyroid cancer incidence patterns in the United States by histologic type, 1992-2006. Thyroid 21: 125-134, 2011.

5. Moley JF: Medullary thyroid carcinoma. Curr Treat Options Oncol 4: 339-347, 2003.

6. Carling T and Udelsman R: Thyroid cancer. Annu Rev Med 65: 125-137, 2014

7. Enewold L, Zhu K, Ron E, Marrogi AJ, Stojadinovic A, Peoples GE and Devesa SS: Rising thyroid cancer incidence in the United States by demographic and tumor characteristics, 1980-2005. Cancer Epidemiol Biomarkers Prev 18: 784-791, 2009.
8. Torchy MP, Hamiche A and Klaholz BP: Structure and function insights into the NuRD chromatin remodeling complex. Cell Mol Life Sci 72: 2491-2507, 2015.

9. Smits AH, Jansen PW, Poser I, Hyman AA and Vermeulen M: Stoichiometry of chromatin-associated protein complexes revealed by label-free quantitative mass spectrometry-based proteomics. Nucleic Acids Res 41: e28, 2013.

10. Brackertz M, Boeke J, Zhang R and Renkawitz R: Two highly related p66 proteins comprise a new family of potent transcriptional repressors interacting with MBD2 and MBD3. J Biol Chem 277: 40958-40966, 2002.

11. Brackertz M, Gong Z, Leers J and Renkawitz R: p66alpha and p66beta of the Mi-2/NuRD complex mediate MBD2 and histone interaction. Nucleic Acids Res 34: 397-406, 2006.

12. Marino S and Nusse R: Mutants in the mouse NuRD/Mi2 component P66alpha are embryonic lethal. PLoS One 2: e519, 2007.

13. Lai AY and Wade PA: Cancer biology and NuRD: A multifaceted chromatin remodelling complex. Nat Rev Cancer 11: 588-596, 2011.

14. Feng Q, Cao R, Xia L, Erdjument-Bromage H, Tempst P and Zhang Y: Identification and functional characterization of the p66/p68 components of the MeCP1 complex. Mol Cell Biol 22: 536-546, 2002

15. Umansky SR, Korol' BA and Nelipovich PA: In vivo DNA degradation in thymocytes of gamma-irradiated or hydrocortisone-treated rats. Biochim Biophys Acta 655: 9-17, 1981.

16. Gong J, Traganos F and Darzynkiewicz Z: A selective procedure for DNA extraction from apoptotic cells applicable for gel electrophoresis and flow cytometry. Anal Biochem 218: 314-319, 1994.

17. Darzynkiewicz Z, Juan G, Li X, Gorczyca W, Murakami T and Traganos F: Cytometry in cell necrobiology: Analysis of apoptosis and accidental cell death (necrosis). Cytometry 27: 1-20, 1997.

18. Nagata S, Nagase H, Kawane K, Mukae N and Fukuyama H: Degradation of chromosomal DNA during apoptosis. Cell Death Differ 10: 108-116, 2003

19. Nagata S: Apoptotic DNA fragmentation. Exp Cell Res 256: 12-18, 2000.

20. Porter AG and Jänicke RU: Emerging roles of caspase-3 in apoptosis. Cell Death Differ 6: 99-104, 1999.

21. Heller B, Wang ZQ, Wagner EF, Radons J, Bürkle A, Fehsel K, Burkart $\mathrm{V}$ and Kolb $\mathrm{H}$ : Inactivation of the poly(ADP-ribose) polymerase gene affects oxygen radical and nitric oxide toxicity in islet cells. J Biol Chem 270: 11176-11180, 1995.

22. Karabay AZ, Aktan F, Sunguroğlu A and Buyukbingol Z: Methylsulfonylmethane modulates apoptosis of LPS/IFN- $\gamma$ activated RAW 264.7 macrophage-like cells by targeting p53, $\mathrm{Bax}, \mathrm{Bcl}-2$, cytochrome $\mathrm{c}$ and PARP proteins. Immunopharmacol Immunotoxicol 36: 379-389, 2014.

23. Kaufmann SH: Induction of endonucleolytic DNA cleavage in human acute myelogenous leukemia cells by etoposide, camptothecin, and other cytotoxic anticancer drugs: A cautionary note. Cancer Res 49: 5870-5878, 1989.

24. Olsen JV, Vermeulen M, Santamaria A, Kumar C, Miller ML, Jensen LJ, Gnad F, Cox J, Jensen TS, Nigg EA, et al: Quantitative phosphoproteomics reveals widespread full phosphorylation site occupancy during mitosis. Sci Signal 3: ra3, 2010.

25. Polo SE, Kaidi A, Baskcomb L, Galanty Y and Jackson SP: Regulation of DNA-damage responses and cell-cycle progression by the chromatin remodelling factor CHD4. EMBO J 29: 3130-3139, 2010. 